\title{
Spin Flipping and Polarization Lifetimes of a 270 MeV Deuteron Beam ${ }^{1}$
}

HTML AEETRFACT + LIIKE I.S. Morozov ${ }^{2 *}$, M.Q. Crawford*, Z.B. Etienne ${ }^{3 *}$, M.C. Kandes*, A.D. Krisch ${ }^{*}$, M.A. Leonova*, D.W. Sivers ${ }^{4 *}$, V.K. Wong*, K. Yonehara*, V.A. Anferov ${ }^{\dagger}$, H.O. Meyer ${ }^{\dagger}$, P. Schwandt ${ }^{\dagger}$, E.J. Stephenson ${ }^{\dagger}$ and B. von Przewoski ${ }^{\dagger}$

${ }^{*}$ Spin Physics Center, University of Michigan, Ann Arbor, MI 48109-1120

${ }^{\dagger}$ Indiana University Cyclotron Facility, Bloomington, IN 47408-0768

\begin{abstract}
We recently studied the spin flipping of a $270 \mathrm{MeV}$ vertically polarized deuteron beam stored in the IUCF Cooler Ring. We swept an rf solenoid's frequency through an rf-induced spin resonance and observed the effect on the beam's vector and tensor polarizations. After optimizing the resonance crossing rate and setting the solenoid's voltage to its maximum value, we obtained a spin-flip efficiency of about $94 \pm 1 \%$ for the vector polarization; we also observed a partial spinflip of the tensor polarization. We then used the rf-induced resonance to measure the vector and tensor polarizations' lifetimes at different distances from the resonance; the polarization lifetime ratio $\tau_{\text {vector }} / \tau_{\text {tensor }}$ was about $1.9 \pm 0.4$.
\end{abstract}

\section{INTRODUCTION}

Polarized beam experiments are now an important part of the programs in many highenergy polarized storage rings. Frequent reversals of the beam polarization direction can greatly reduce the detectors' systematic errors in spin asymmetry measurements. Artificial rf-induced spin resonances can be used to do such reversals, or spin-flips, in a very-well-controlled way. We have successfully spin-flipped beams of spin- $\frac{1}{2}$ particles: a horizontally polarized electron beam at the MIT-Bates Storage Ring [1], and horizontally and vertically polarized proton beams at the IUCF Cooler Ring [2, 3]. We recently studied the more complex spin-flipping of a polarized deuteron beam stored in the IUCF Cooler Ring.

In any flat circular accelerator or storage ring, each deuteron's spin precesses around the Stable Spin Direction, which is defined by the Ring's magnetic structure. With no horizontal magnetic fields present in the Ring, the Stable Spin Direction points along the vertical field direction of the Ring's dipole magnets. Moreover, the spin tune $v_{s}$, which is the number of spin precessions during one turn around the Ring, is proportional to the

\footnotetext{
${ }^{1}$ Supported by research grants from the U.S. Department of Energy and the U.S. National Science Foundation

2 E-mail: morozov@umich.edu

3 Also at: Indiana University Cyclotron Facility, Bloomington, IN 47408-0768

${ }^{4}$ Also at: Portland Physics Institute, Portland, OR 97201, USA
} 


$$
v_{s}=G \gamma,
$$

where $G=(g-2) / 2=-0.1426$ is the deuteron's gyromagnetic anomaly and $\gamma$ is its Lorentz energy factor.

The deuteron's vertical polarization can be perturbed by a horizontal rf magnetic field from either an rf-solenoid or an rf-dipole magnet. This perturbation can induce an rf spin resonance, which can be used to flip the spin of the stored polarized deuterons $[2,3]$. The frequency $f_{r}$, at which an rf-induced spin resonance occurs, is given by

$$
f_{r}=f_{c}\left(k \pm v_{s}\right),
$$

where $f_{c}$ is the deuterons's circulation frequency and $k$ is an integer. When an rf solenoid operates at the resonance frequency, its spin rotations accumulate coherently after each turn around the Ring; this can cause large deviations of the polarization from the Stable Spin Direction, which depolarize the beam.

However, sweeping the rf magnet's frequency through $f_{r}$ can rotate the Ring's Stable Spin Direction by $180^{\circ}$ with no depolarization. If the rotation is adiabatic, then each deuteron's vertical spin follows the Stable Spin Direction as it rotates by $180^{\circ}$ resulting in a polarization flip. We use a modified [1] Froissart-Stora formula [4] to relate the beam's initial vector polarization $P_{i}$, to its final vector polarization $P_{f}$, after crossing the resonance,

$$
\frac{P_{f}}{P_{i}}=(1+\eta) \exp \left[\frac{-\left(\pi \varepsilon f_{c}\right)^{2}}{\Delta f / \Delta t}\right]-\eta,
$$

where $\eta$ is the spin-flip efficiency, $\varepsilon$ is the resonance strength, and $\Delta f / \Delta t$ is the resonance crossing rate, while $\Delta f$ is the frequency range during the ramp time $\Delta t$.

The polarization of a beam of spin-1 particles is more complex than that of spin- $\frac{1}{2}$ particles; the component of the spin along the vertical axis can have three values: $m_{z}=+1,0,-1$; moreover, a spin-1 particle is also usually parameterized by a quantity called the tensor polarization [5]

$$
p_{z z} \equiv 1-3\left(N_{0} / N_{T}\right)
$$

where $N_{0}$ is the number of particles in the $m_{z}=0$ state and $N_{T}$ is the total number of particles. Eq. (4) is defined so that an unpolarized beam with all $m_{z}$ states equally populated, has $p_{z z}=0$. To describe a change in the tensor polarization after a frequency ramp we developed Eq. (5),

$$
\frac{P_{z z f}}{P_{z z i}}=\frac{3}{2}\left\{(1+\eta) \exp \left[\frac{-\left(\pi \varepsilon f_{c}\right)^{2}}{\Delta f / \Delta t}\right]-\eta\right\}^{2}-\frac{1}{2},
$$

which is an extension of the Froissart-Stora formula [4] to tensor polarization using Eq. (4). 


\section{Experimental Apparatus}

The apparatus used for this experiment, including the rf-solenoid, the IUCF Cooler Ring and the polarimeter are shown in Fig. 1. For this experiment an unpolarized hydrogen gas cell was used as polarimeter's target. The thickness of the gas target was optimized to about $5 \cdot 10^{13}$ atoms $/ \mathrm{cm}^{2}$ to maximize the statistics while minimizing the background due to both the cell walls and deuteron breakup.

The $270 \mathrm{MeV}$ polarized deuteron beam stored in the Cooler Ring was obtained using the new Cooler Injector Polarized IOn Source (CIPIOS) and the new Cooler Injection Synchrotron (CIS). To reduce the systematic errors in our polarization measurements, we normally cycled the deuteron beam through the four vertical polarization states available in CIPIOS: $\left|p_{z} p_{z z}\right\rangle$ of $|11\rangle,|-11\rangle,|01\rangle$ and $|0-2\rangle$, and occasionally through the totally unpolarized state $|00\rangle$ to check systematic errors.

The vector polarization's magnitude in the Cooler Ring, was about 0.6 for the $p_{z}: \pm 1$ states; the tensor polarization magnitudes, for the $p_{z z}:+1$ and $p_{z z}:-2$ states, were about 0.8 and -1.6 , respectively. Each data point required about an hour to obtain statistical errors of about $\pm 2 \%$ for the vector polarization and about $\pm 5 \%$ for the tensor polarization.

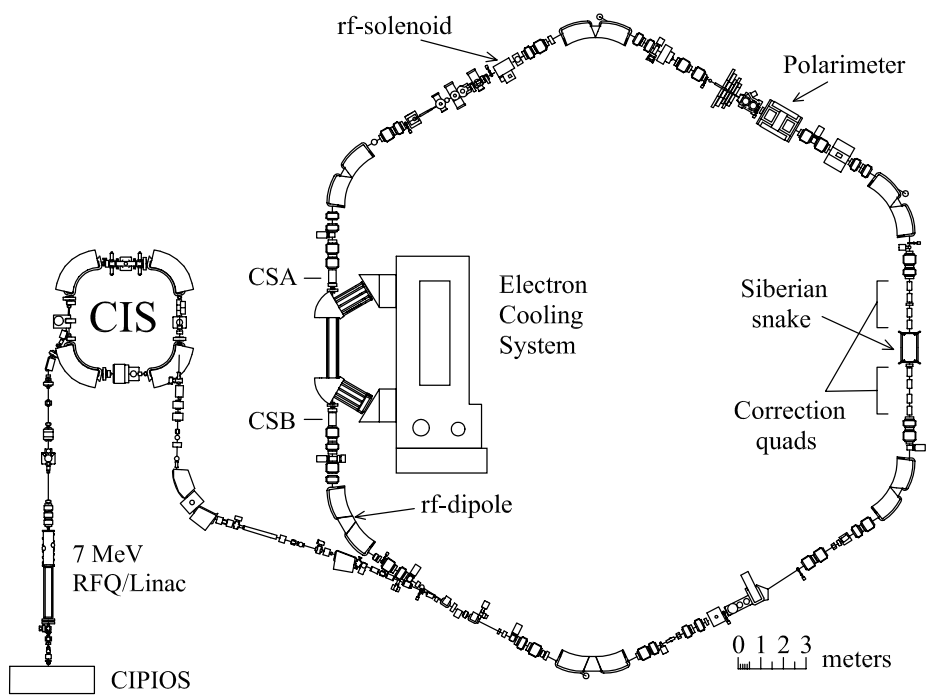

FIGURE 1. Layout of the IUCF Cooler Ring with its new Cooler Injector Synchrotron (CIS) and its new CIPIOS polarized ion source. Also shown inthe Cooler Ring are the rf-dipole, the rf-solenoid, the polarimeter, and the Siberian snake. 


\section{Experimental Results (February 2002)}

We first found the approximate position of the $f_{r}=f_{c}\left(1-\left|v_{s}\right|\right)$ spin depolarizing resonance by sweeping the rf solenoid's frequency first over $64 \mathrm{kHz}$ and then narrowing the frequency range into those ranges, which caused spin flip. We then more precisely determined $f_{r}$ by measuring the vertical polarization after running the $\mathrm{rf}$ solenoid at different fixed frequencies. From these data we found that the resonance frequency was $f_{r}=1403002 \pm 14 \mathrm{~Hz}$ for the vector plarization and $f_{r}=1402999 \pm 17 \mathrm{~Hz}$ for the tensor polarization.

We next optimized the rf-solenoid's frequency ramp time $\Delta t$ and frequency range $\Delta f$ to maximize the spin-flip efficiency for the deuteron's vector polarization. We first linearly ramped the rf-solenoid's frequency from $f_{r}-2$ to $f_{r}+2 \mathrm{kHz}$, with various ramp times $\Delta t$, while measuring the polarization after each frequency ramp. The measured vector and tensor polarizations are plotted against the ramp time in Fig. 2. The vector polarization data are fit to Eq. (3) by ignoring the $\Delta t=500 \mathrm{~ms}$ point, which seems anomalous. The tensor polarization data in Fig. 2 are fit to Eq. (5) as shown by the curves. Next, using the optimum $\Delta t$ of $2 \mathrm{~s}$, we spin-flipped the deuterons while varying the rf solenoid's frequency range $\Delta f$. These studies gave an optimum $\Delta f$ of $\pm 0.75 \mathrm{kHz}$.

After setting $\Delta t, \Delta f$ and the voltage to maximize the vector polarization spin-flip efficiency $\eta$, we more precisely determined $\eta$ by measuring the vector polarization while varying the number of frequency sweeps. This measured vector and tensor polarization after $n$ frequency sweeps are each plotted against $n$ in Fig. 3. We fit these data using

$$
P_{n}=P_{i} \cdot \eta^{n}
$$

where $P_{n}$ is the measured radial beam polarization after $n$ spin flips. The fits gave a vector polarization spin-flip efficiency of $94 \pm 1 \%$ and a tensor depolarization of $18 \pm 3 \%$ per frequency ramp.

We also studied the polarizations' lifetimes near the resonance. We turned the rf solenoid on at a fixed frequency near the depolarizing resonance and observed the polarization's decay with time. From these data we extracted lifetimes of the vector and tensor polarization at three distances from the resonance. Then we calculated the

polarization lifetime ratio $\tau_{\text {vector }} / \tau_{\text {tensor }}$ to be about $1.9 \pm 0.4$.

We would like to thank the entire Indiana University Cyclotron Facility staff for the successful operation of the Cooler Ring

\section{REFERENCES}

1. V.S. Morozov et al. Phys. Rev. ST-AB 4, 104002 (2001).

2. D.D. Caussyn et al., Phys. Rev. Lett. 73, 2857 (1994).

3. B.B. Blinov et al., Phys. Rev. Lett. 88, 014801 (2002).

4. M. Froissart and R. Stora, Nucl. Instrum. and Methods 7, 297 (1960).

5. The Madison Convention, Proc. of 3rd Intl. Symp. on Polarization Phenomena in Nuclear Physics, Madison, 1970, the University of Wisconsin Press, p. xxv (1971). 

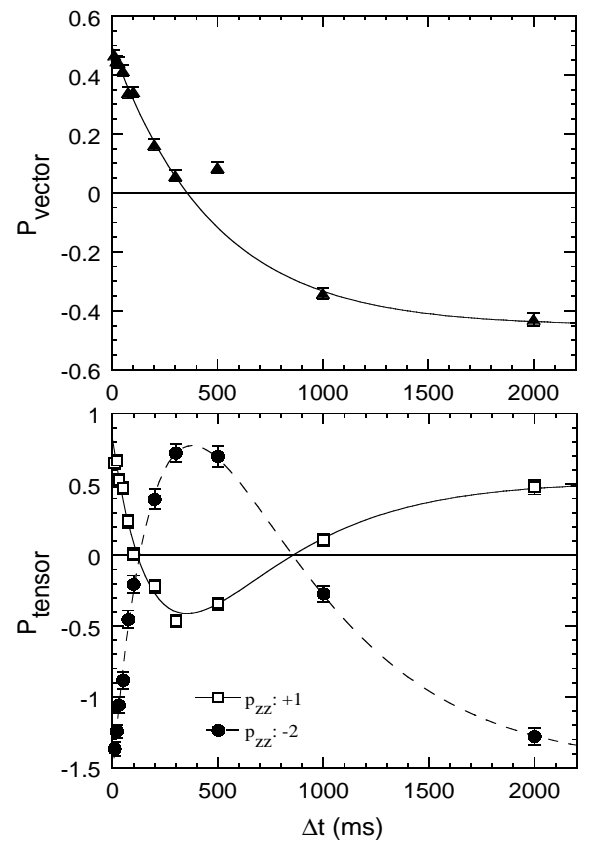

FIGURE2. The measured vector and two tensor deuteron polarizations at $270 \mathrm{MeV}$ are plotted against the rf solenoid ramp time $\Delta t$. The curve in the $P_{\text {vector }}$ plot is a fit using Eq. (3). The solid and dashed lines in the bottom plot are fits to the $P_{\text {tensor }}$ data using Eq. (5).

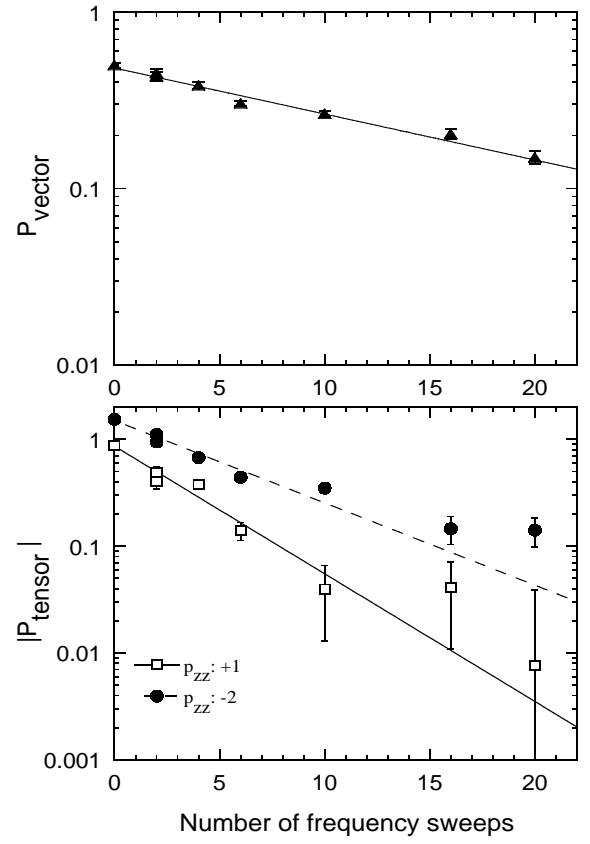

FIGURE3. The measured vector and two tensor deuteron polarizations at $270 \mathrm{MeV}$ are plotted against the number of frequency sweeps. The solid and dashed curves are fits to Eq. (6). 